\title{
Preserving Radio Astronomy in Developing Nations
}

\author{
G. Swarup and C. R. Subramanya \\ National Centre for Radio Astrophysics, \\ Tata Institute of Fundamental Research Poona University Campus, \\ Pune - 411007 India. \\ gswarup@gmrt.ernet.in
}

Raman Research Institute, C. V. Raman Avenue Sadashivnaga, Bangalore, Karnataka 560 080, India.

\begin{abstract}
Due to the very weak nature of signals from cosmic radio sources, the sensitivity of a radio telescope and receiver is about 40$60 \mathrm{~dB}$ higher than those of communications receivers. Hence, radio telescopes are generally located in relatively radio-quiet locations and operate in frequency bands that are protected against radio interference through frequency planning by national governments. Taking advantage of the much lower degree of radio interference in developing countries and the relatively labour-intensive nature of metre-wave radio telescopes, several such radio telescopes have been built and are planned in Argentina, Brazil, China, India, Mauritius and South Africa. Radio telescopes operating at $\mathrm{cm}$-wavelengths are also planned in Egypt and Mexico.

A particularly severe problem arises for the radio astronomy service and other passive services below $2 \mathrm{GHz}$ from the possibility of unacceptable emissions from satellites in unwanted bands (out-of-band and spurious emissions), due to the specific modulation schemes used in satellite transmitters. It is noted that this can be circumvented within the existing technologies if the satellite transmitters employ suitable bit-shaping or filtering techniques or use modulation schemes like Gaussian-filtered Minimum-Shift Keying (GMSK) which produce very little out-of-band emission. Although radio astronomy started in the western world at low frequencies, much low frequency radio astronomy is now planned or operational in developing countries. In order to protect the interests of these and other passive services within developing nations, it is important that suitable regulations be recommended to UNISPACE-III to provide appropriate protection.
\end{abstract}

